\title{
Effect of Curcumin on Hippocampal Levels of Brain-Derived Neurotrophic Factor and Serum Levels of Inflammatory Cytokines in Rat Model for Alzheimer's Disease
}

\author{
Seyed Damoon Sadoughi ${ }^{*}$, Jina Khayatzadeh ${ }^{2}$ \\ ${ }^{1}$ Young Researchers and Elite Club, Mashhad Branch, Islamic Azad University, Mashhad, Iran \\ ${ }^{2}$ Department of Biology, Faculty of Sciences, Mashhad Branch, Islamic Azad University, Mashhad, Iran
}

\section{A BSTRACT}

Introduction: Alzheimer's disease (AD) is a progressive neurodegenerative disease of the central nervous system. Neuronal injury and oxidative stress in AD increases the level of inflammatory cytokines. Curcumin has antioxidant and neuroprotective properties. The aim of this study was to determine the effect of curcumin on hippocampal levels of brain-derived neurotrophic factor (BDNF) and serum levels of inflammatory cytokines in an experimental model of AD. Materials and Methods: 28 male rats divided into 4 groups: Control group (30 days, intraperitoneal injection of DMSO), AD control group (30 days, intraperitoneal injection of DMSO, after induction of $\mathrm{AD}$ ) and two $\mathrm{AD}$ treatment groups with application of 50 and $100 \mathrm{mg} / \mathrm{kg}$ of curcumin (30 days, intraperitoneal injection of curcumin, after induction of $\mathrm{AD}$ ). $\mathrm{AD}$ was induced by intraperitoneal injection of $8 \mathrm{mg} / \mathrm{kg}$ trimethyltin chloride. At the end of the treatment period, the hippocampal levels of BDNF and serum levels of different cytokines (TNF- $\alpha$, IL-1 $\beta$ and IL-6) were measured by ELISA method. Results: Compared to AD control group, administration of curcumin with dose of $100 \mathrm{mg} / \mathrm{kg}$ significantly increased the hippocampal levels of $\operatorname{BDNF}(\mathrm{P}=0.002)$ and decreased the serum levels of TNF- $\alpha$, IL-1 $\beta$ and IL-6 $(\mathrm{P}=0.001)$. Conclusion: Administration of curcumin may decrease the serum levels of inflammatory cytokines in AD, possibly via enhancement of the hippocampal levels of BDNF. This study suggests the protective effect of curcumin against neuronal damages and oxidative stress in AD.

\section{Key words:}

1. Curcumin

2. Rats

3. Brain-Derived

Neurotrophic Factor

*Corresponding Author: Seyed Damoon Sadoughi

E-mail: Damoon.sadoughi@mshdiau.ac.ir 


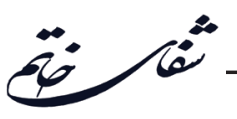

اثر كوركومين بر سطح هيبوكاميى فاكتور نوروتروفيك مشتق از مغز و سطح سرمى سايتوكينهاى

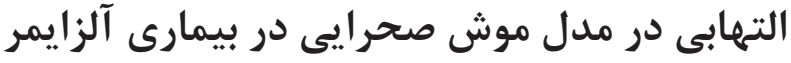

\author{
سيد دامون صدوقى '“:، جينا خياطزاده广 \\ 'باشكاه يُروهشكَران جوان و نخبكان، واحد مشهد، دانشكاه آزاد اسلامى، مشهد، ايران

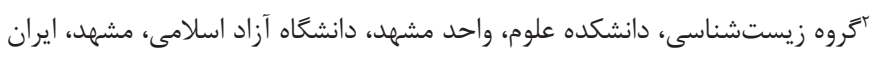

اطلاعات مقاله:

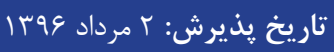

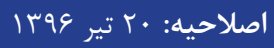

تاريخ دريافت: وץ فروردين عوسا

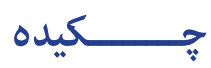

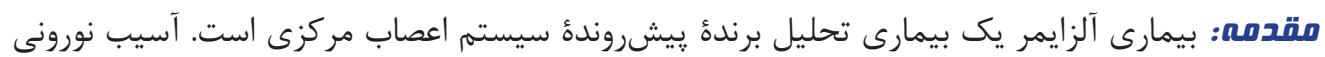

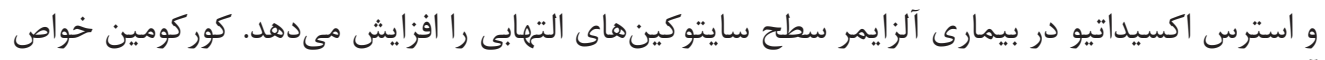

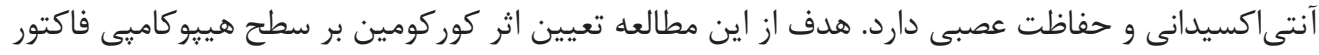

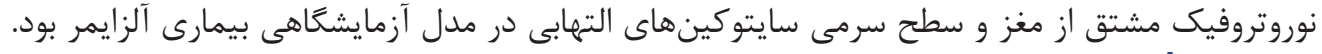

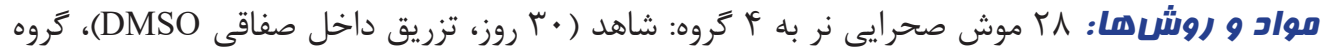

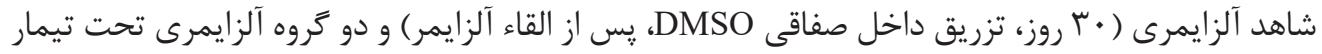

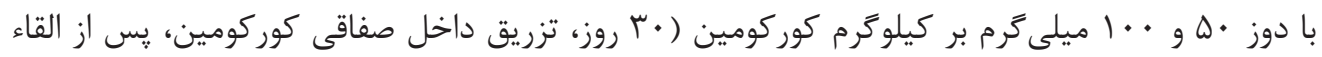

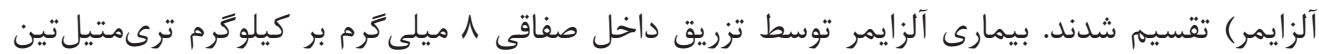

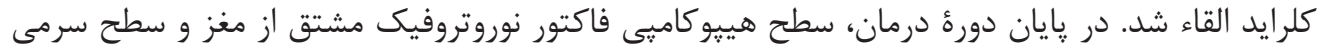
سايتوكينهاى مختلف (TNF-

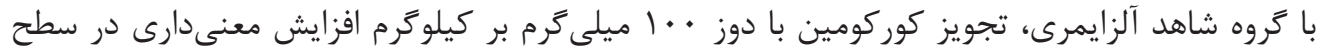

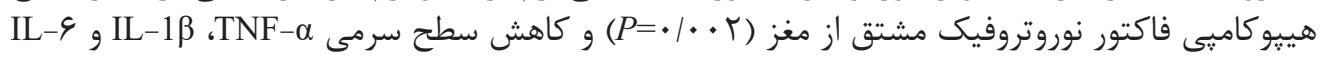
التهابي (•l)

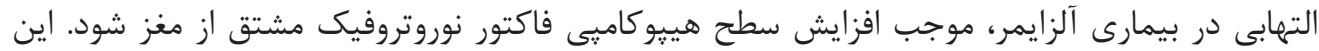

كليد وازهها:

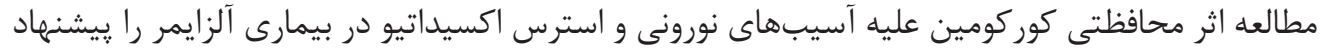

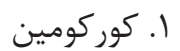

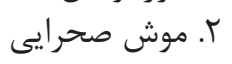

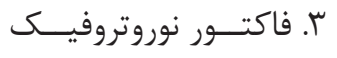

مشـتق از منــن مى مدهد.

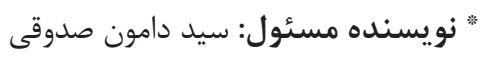
آدرس الكترونيكى: Damoon.sadoughi@mshdiau.ac.ir 


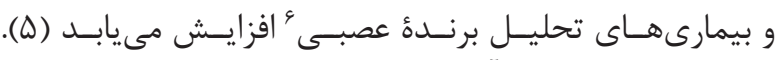

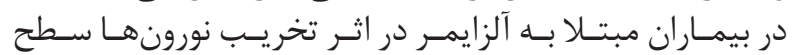

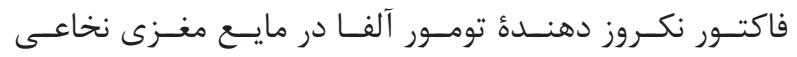

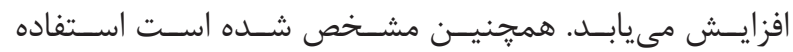

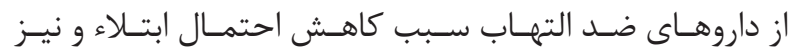

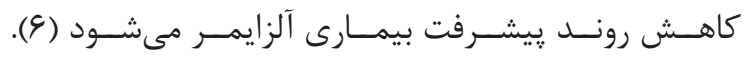

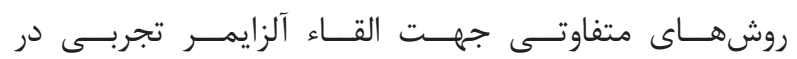

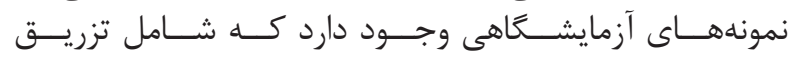

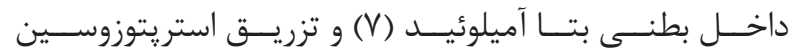

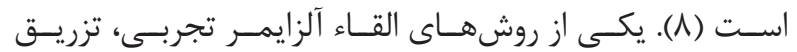

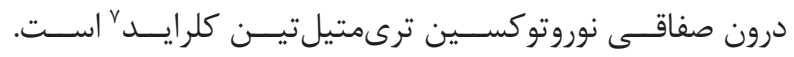

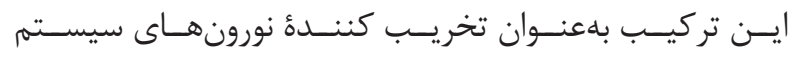

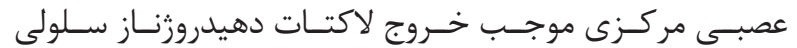

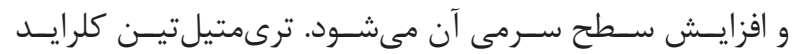

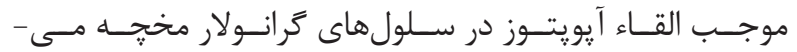

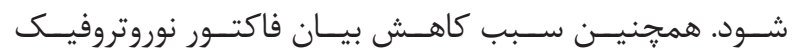

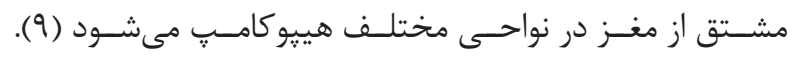

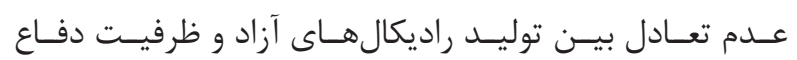

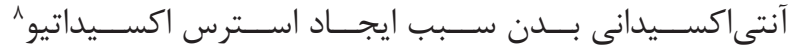

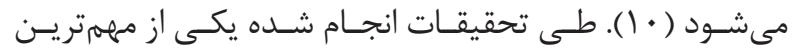

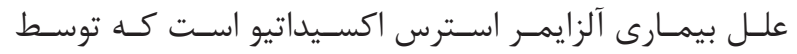

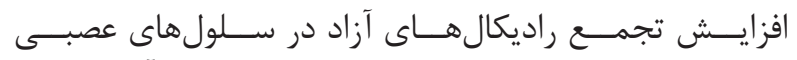

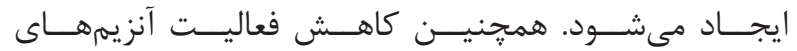

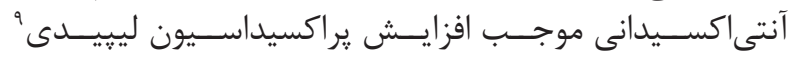

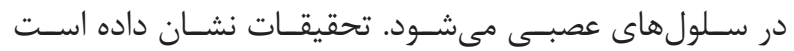

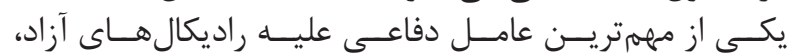

آنتى اكســـيدانها هســتند (1) (1).

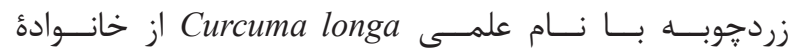

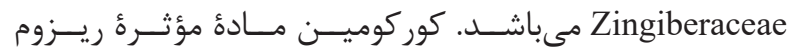

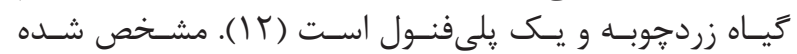

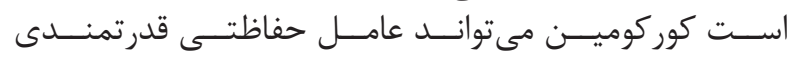

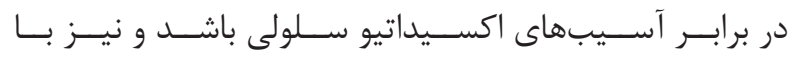

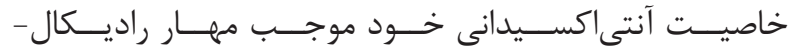

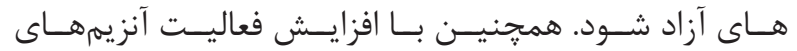

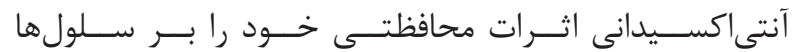

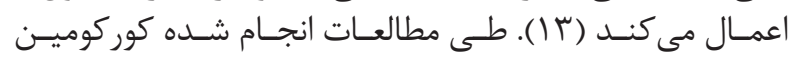

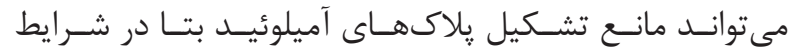

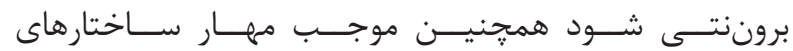

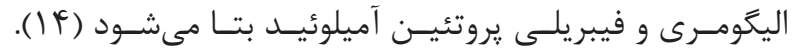

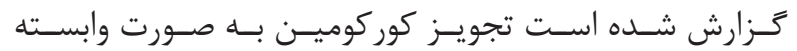

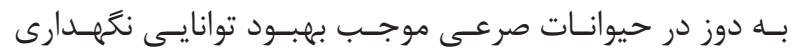

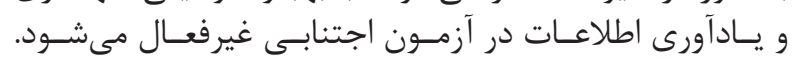

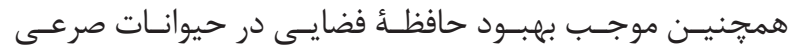

${ }^{1}$ Nucleus basalis of meynert

${ }^{2}$ Nerve growth factor

${ }^{3}$ Brain-derived neurotrophic factor

${ }^{4}$ Neurotrophin-3

${ }^{5}$ Tumor necrosis factor-alpha

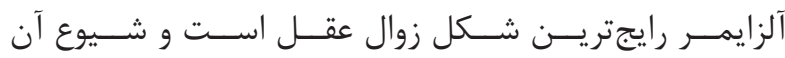

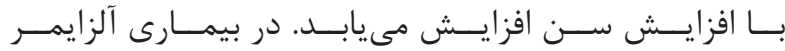

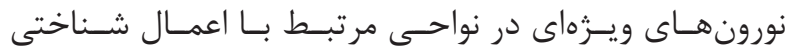

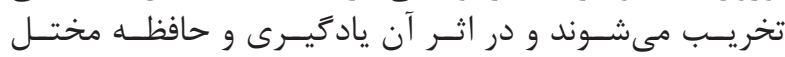

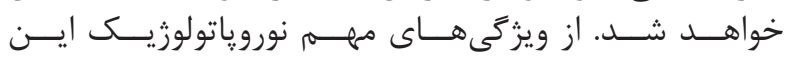

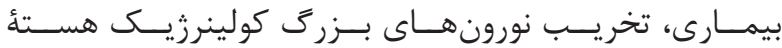

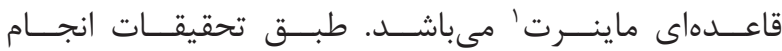

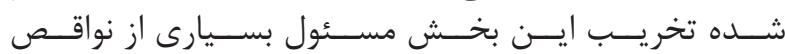

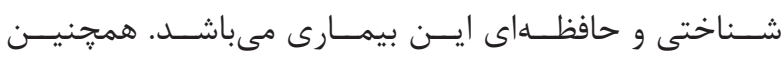

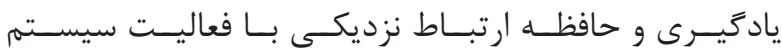

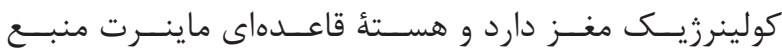

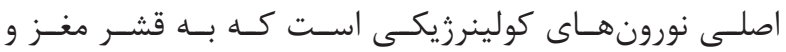

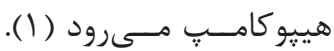

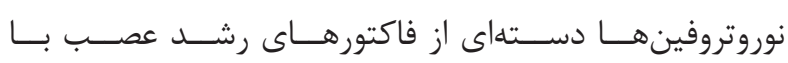

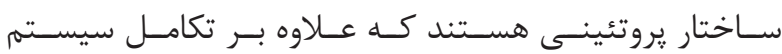

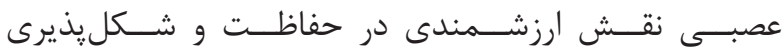

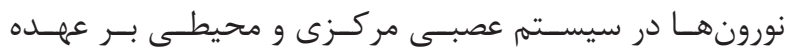

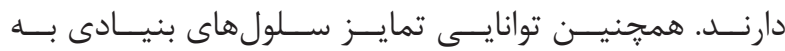

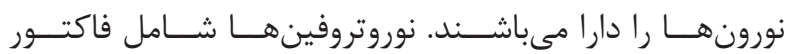

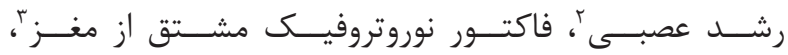

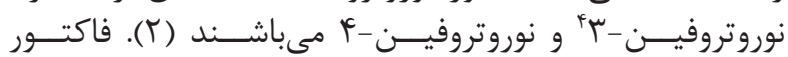

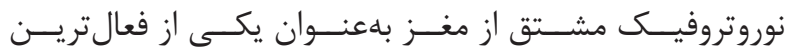

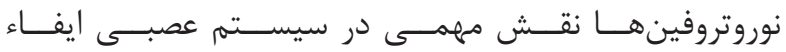

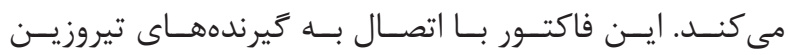

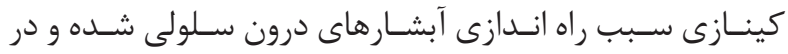

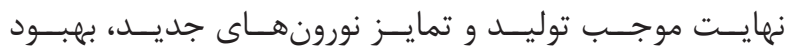

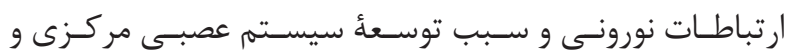

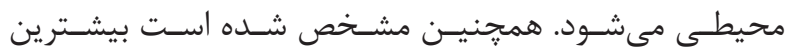

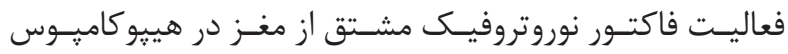

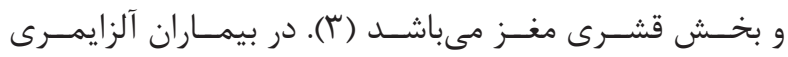

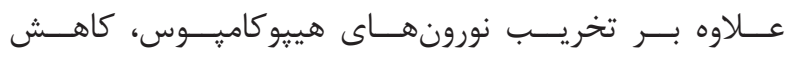

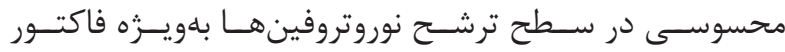

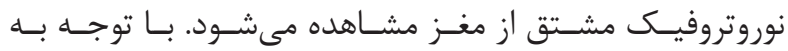

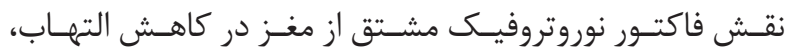

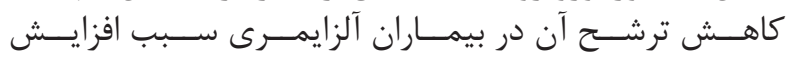

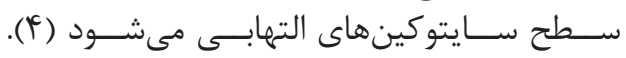

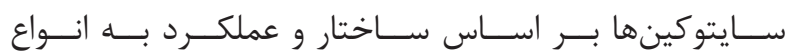

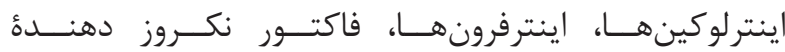

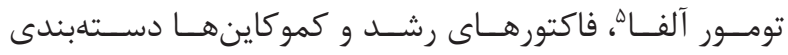

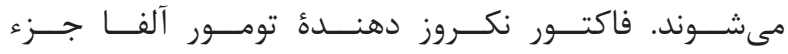

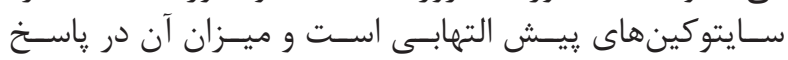

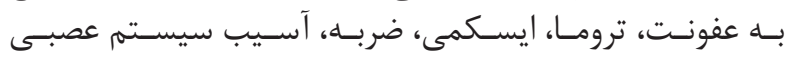

${ }^{6}$ Neurodegenerative diseases

${ }^{7}$ Trimethyltin chloride

${ }^{8}$ Oxidative stress

${ }^{9}$ Lipid peroxidation 


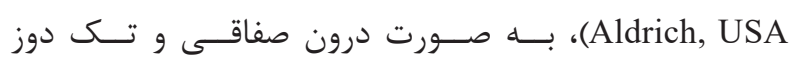

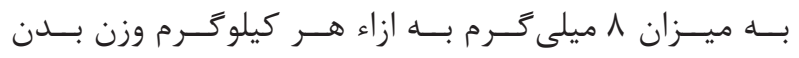

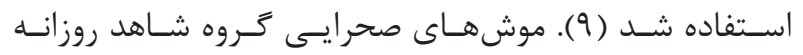

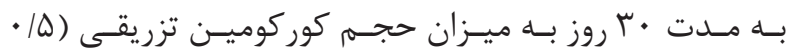

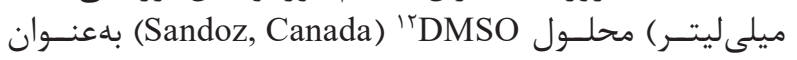

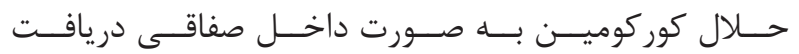

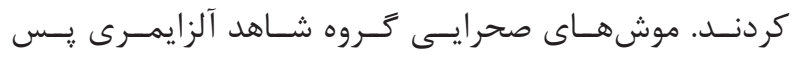

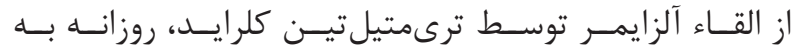

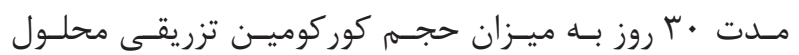

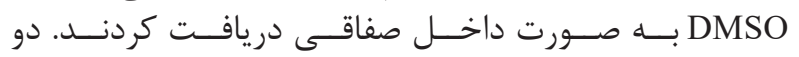

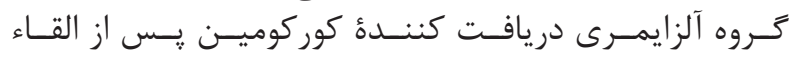

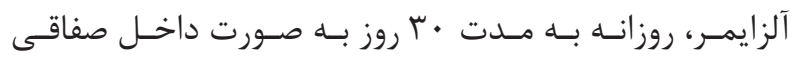

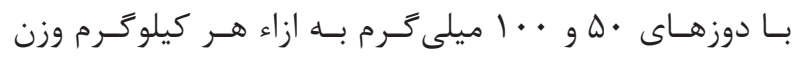
بـدن تيمــار شــدند.

\section{سنجش هاى بيوشيميايى}

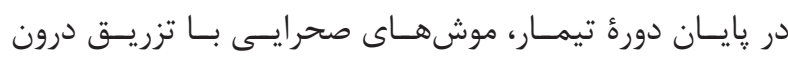

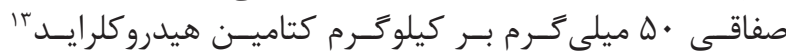
و ه ه ميلى

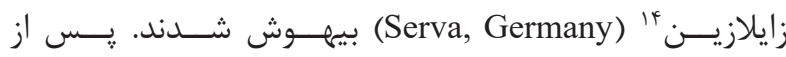

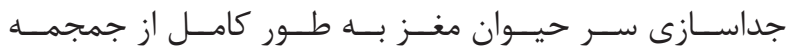

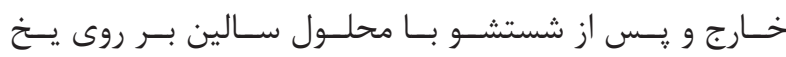

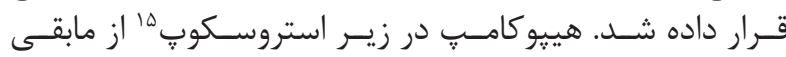

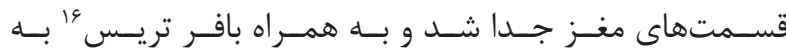

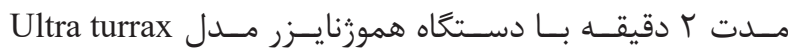
(IKA, Germany) T25 digital

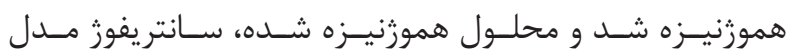

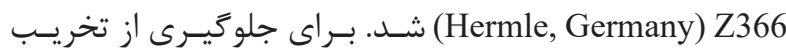

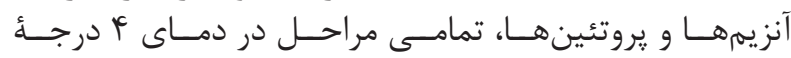

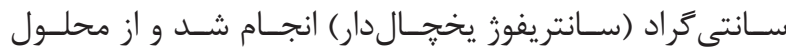

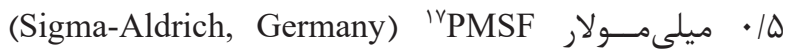

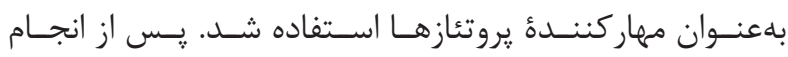

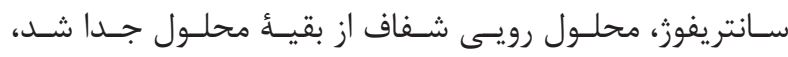

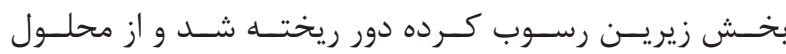

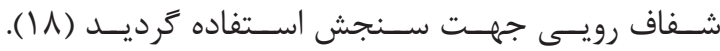

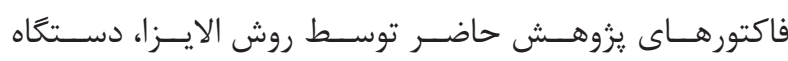

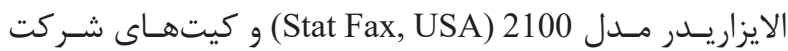

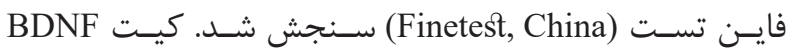

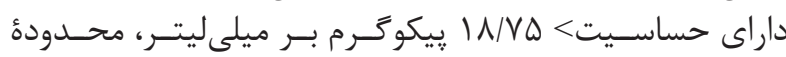

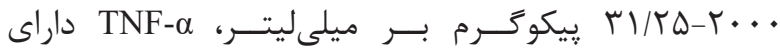

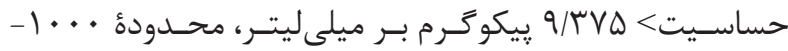

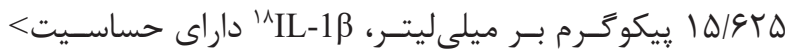
IN/VD

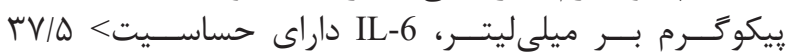

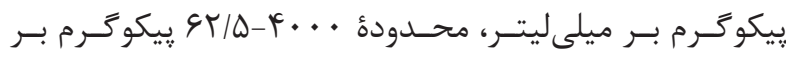

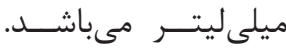

\footnotetext{
${ }^{10}$ Neuroprotective

${ }^{11}$ Wistar

${ }^{12}$ Dimethyl sulfoxide

${ }^{13}$ Ketamine hydrochloride

${ }^{14}$ Xylazine
}

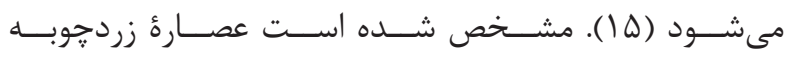

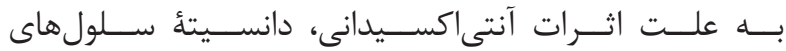

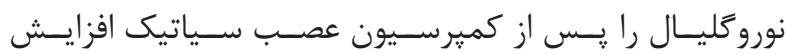

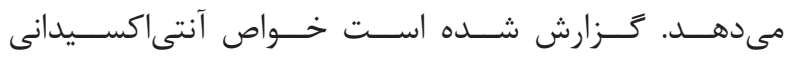

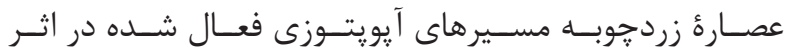

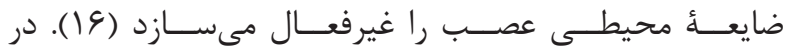

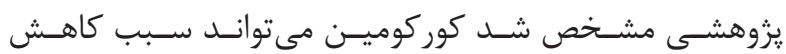

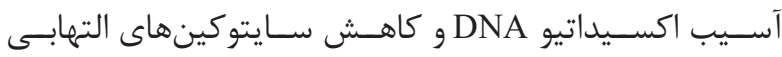

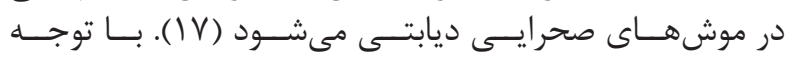

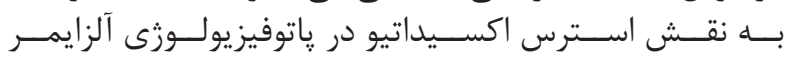

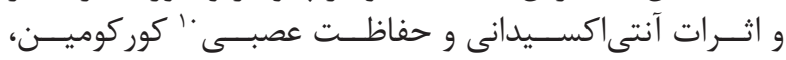

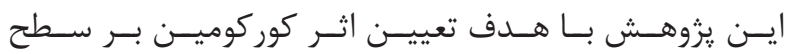

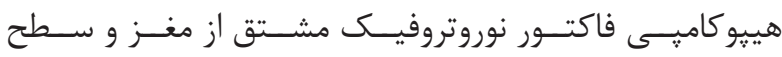

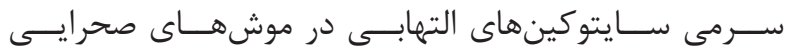

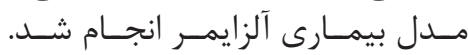

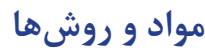
حيوانات و گروههاى آزمايشى

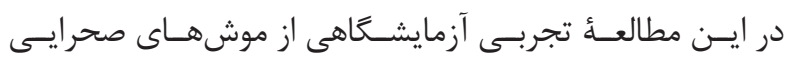

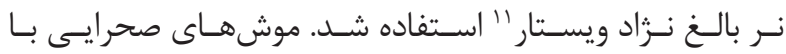

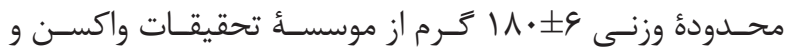

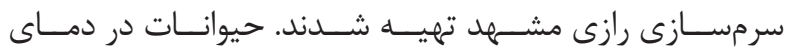

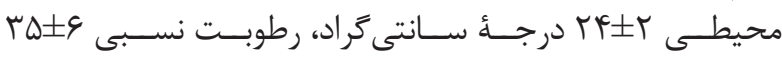

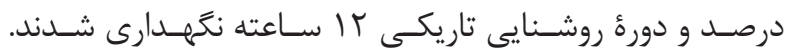

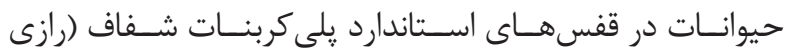

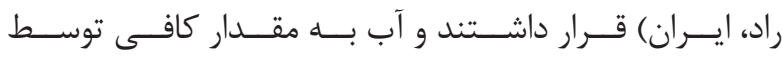

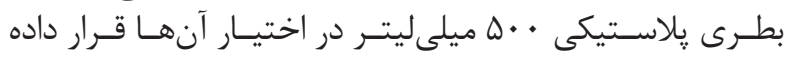

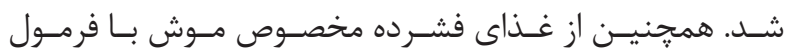

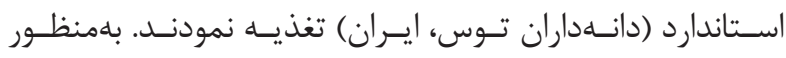

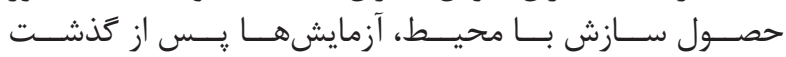

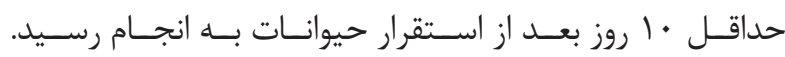

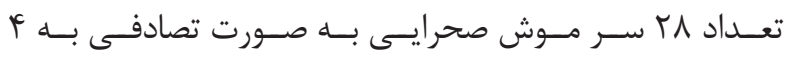

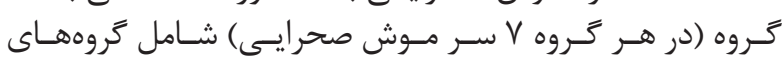

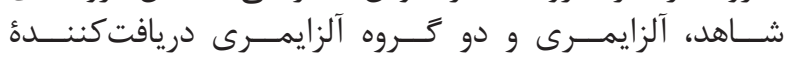

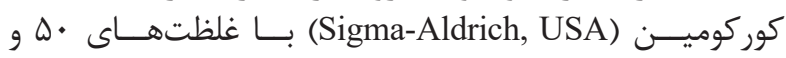

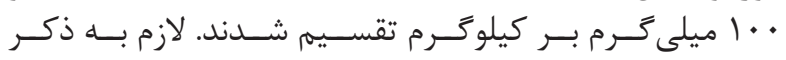

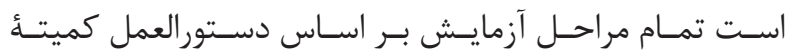

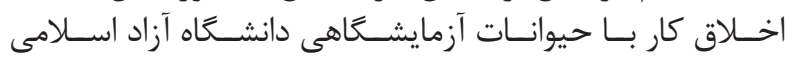

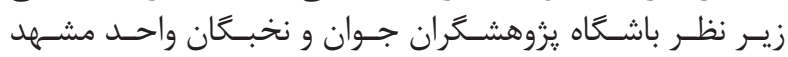

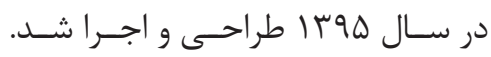

القاء مدل حيوانى آلزايمر و تيمارها

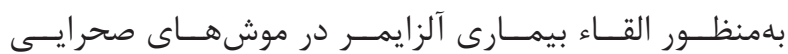

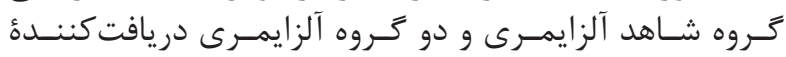

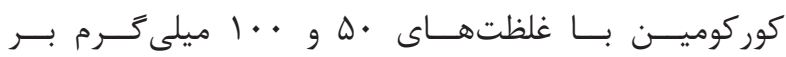

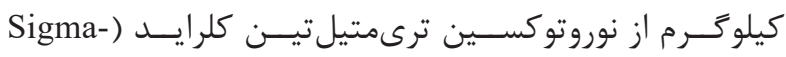
${ }^{15}$ Stereoscope
${ }^{16}$ Tris buffer
${ }^{17}$ Phenylmethylsulfonyl fluoride
${ }^{18}$ Interleukin- $1 \beta$ 


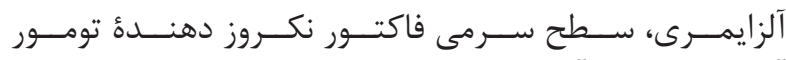

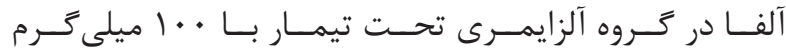

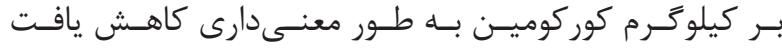

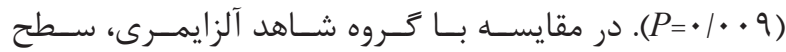

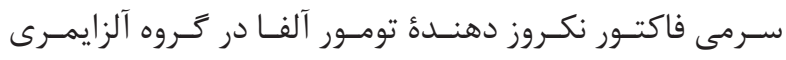

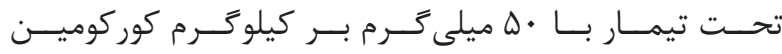

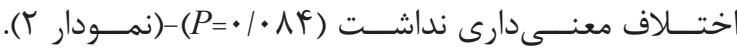

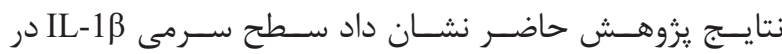

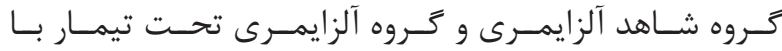

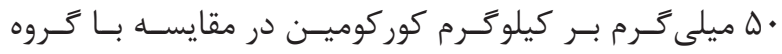

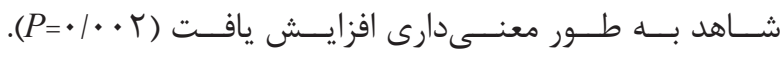

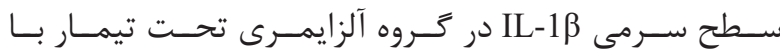

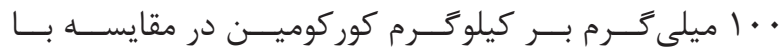

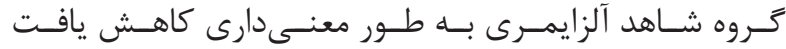
تيمار

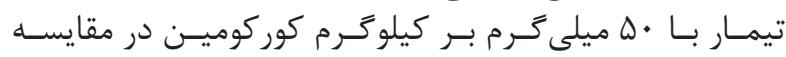

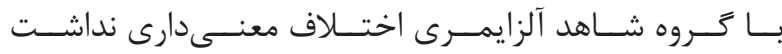

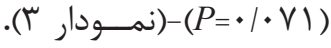

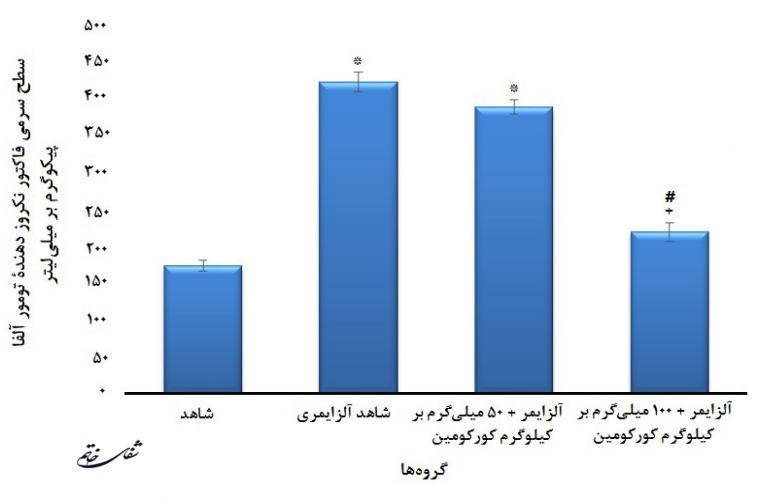

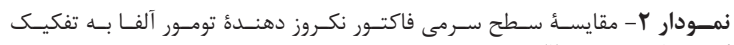

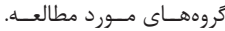

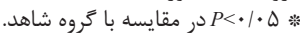

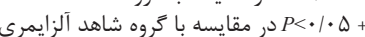

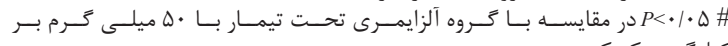
كيلوخَــرم كوركوميـنـن

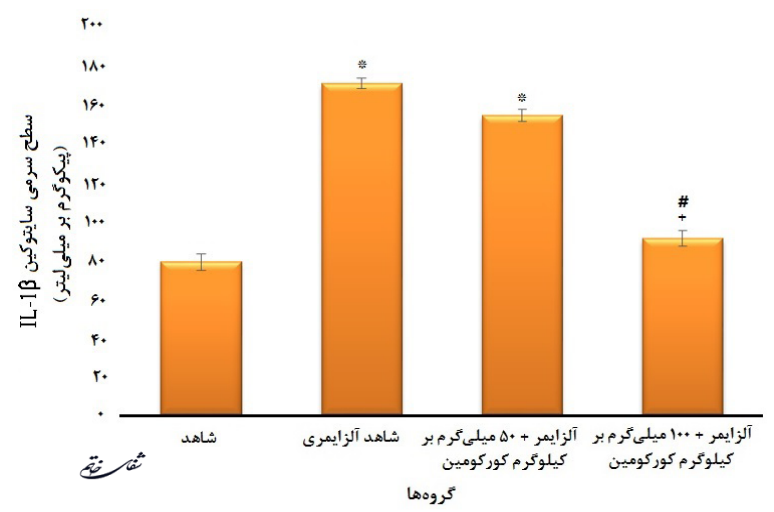

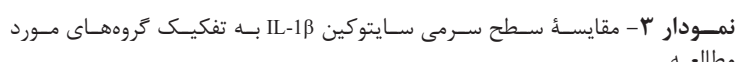
P م مقايسه با گروه شاهد.

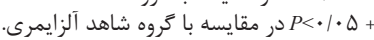

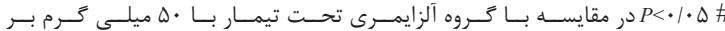

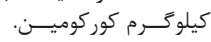

آناليز آمارى

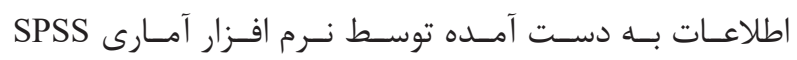

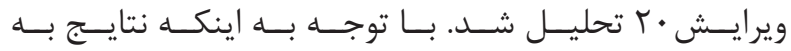

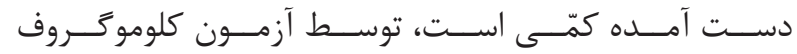

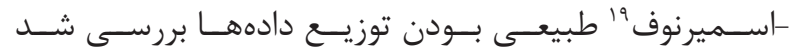

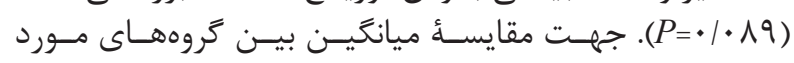

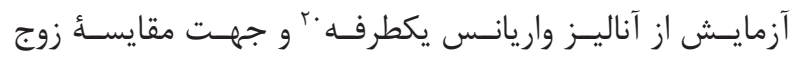

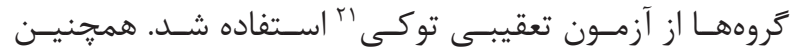

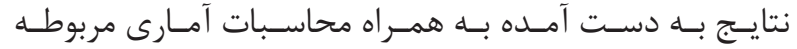

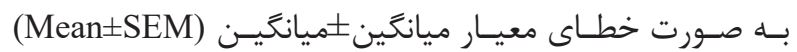

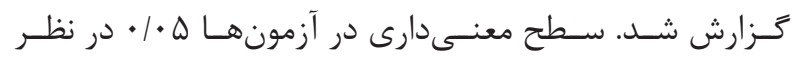

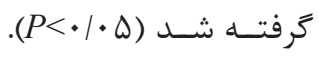

يافتهها

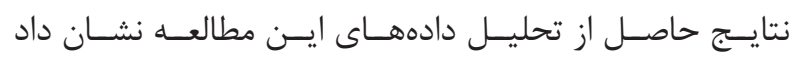

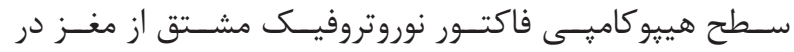

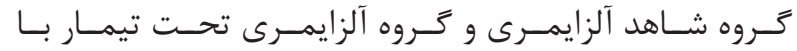

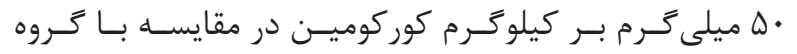

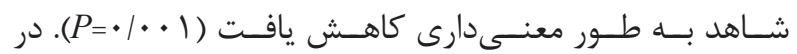

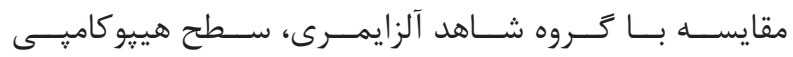

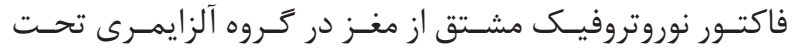

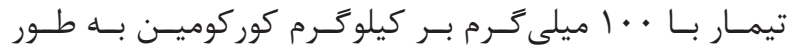

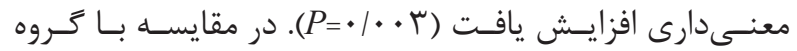

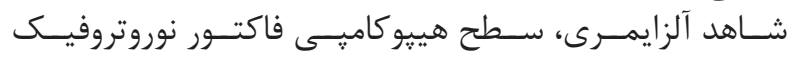

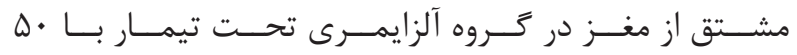

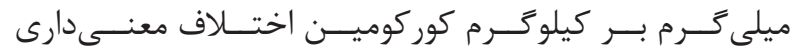

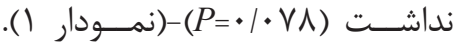

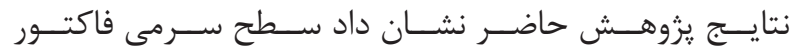

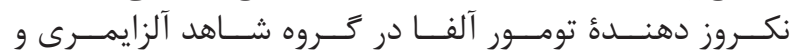

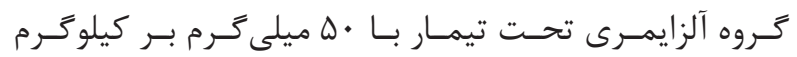

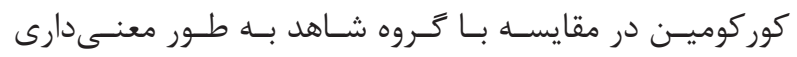

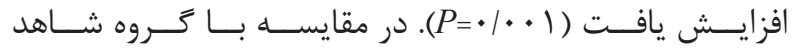

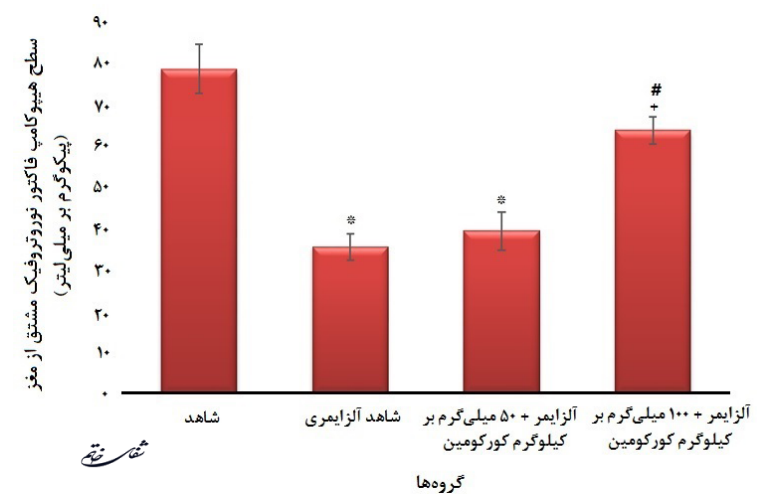

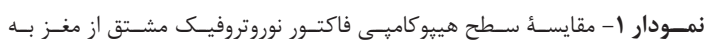

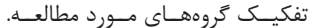

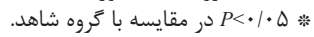

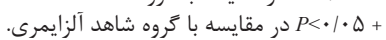

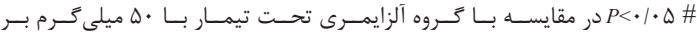
كيلوگتــرم كور كوميـن.

${ }^{19}$ Kolmogorov-Smirnov

${ }^{20}$ One-way ANOVA

${ }^{21}$ Tukey 


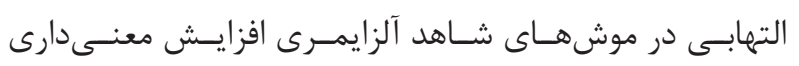

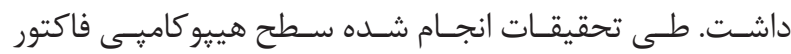

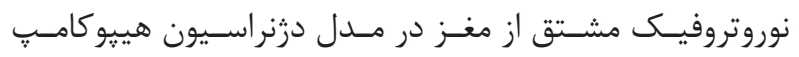

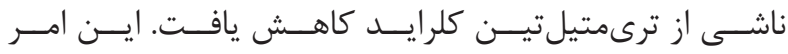

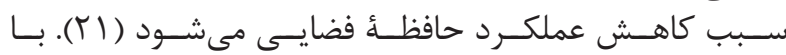

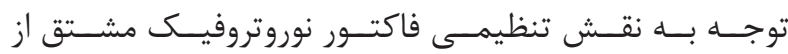

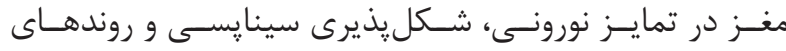

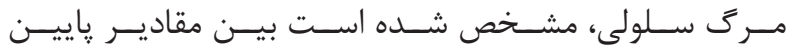

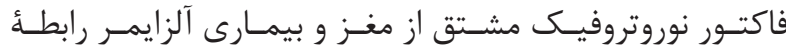

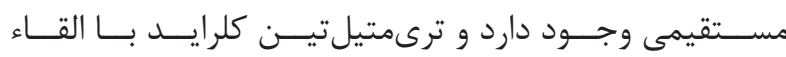

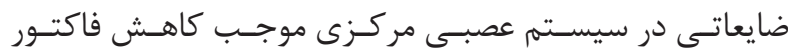

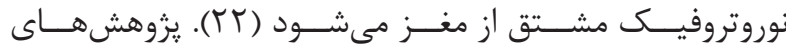

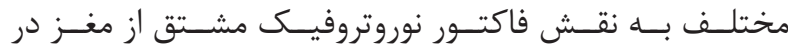

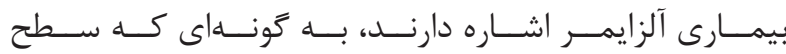

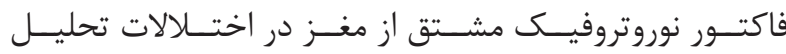

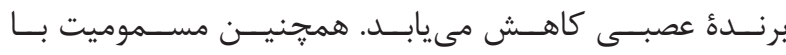

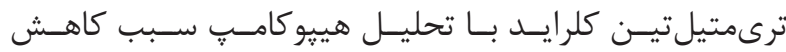

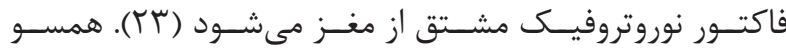

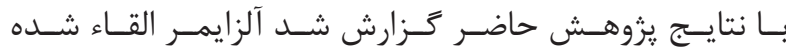

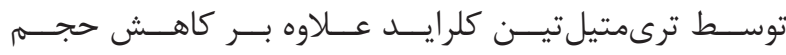

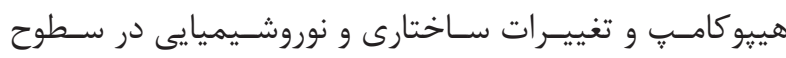

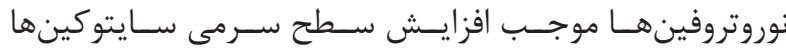

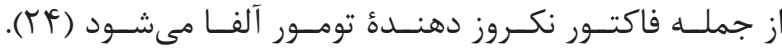

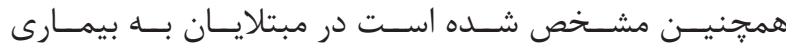

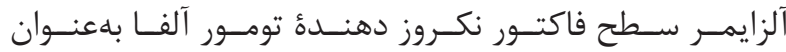

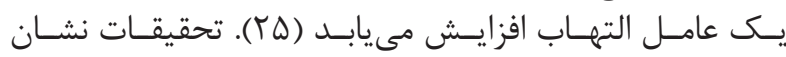

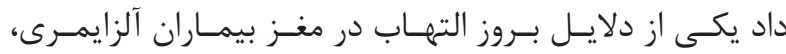

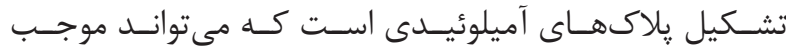

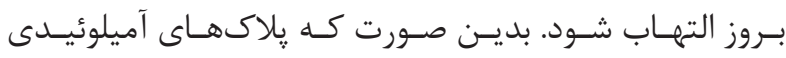

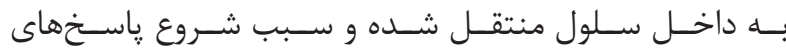

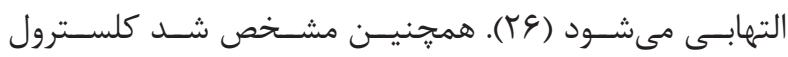

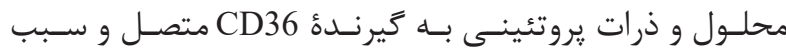

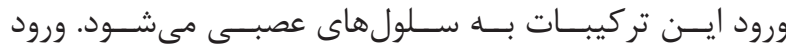

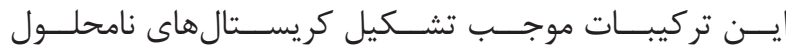

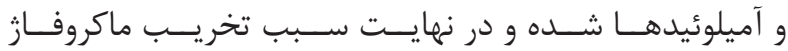

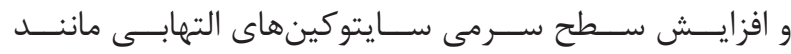

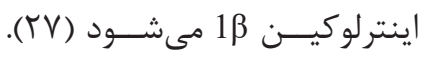

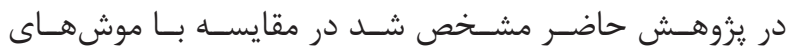

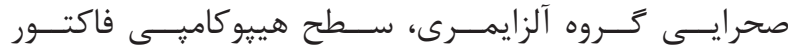

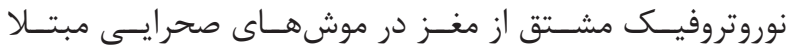

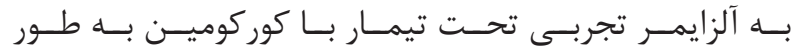

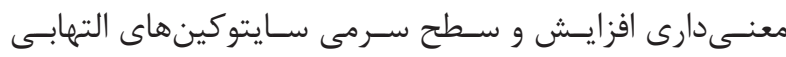

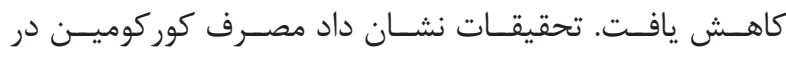

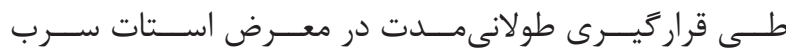

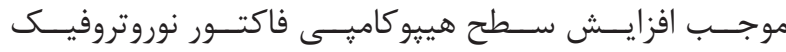

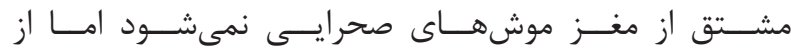

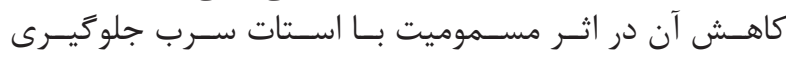

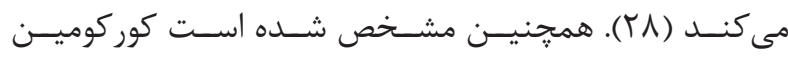

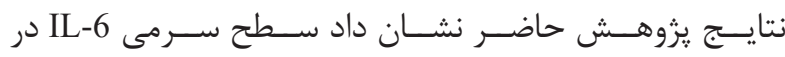

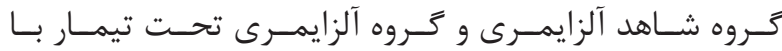

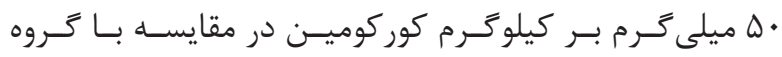

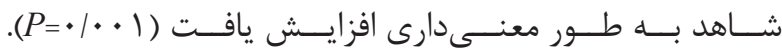

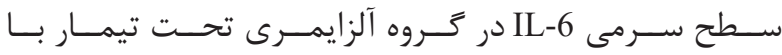

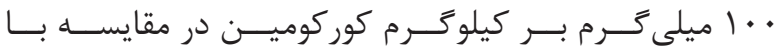

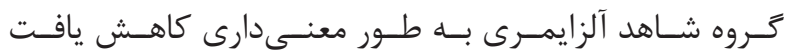

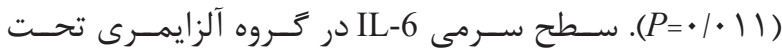

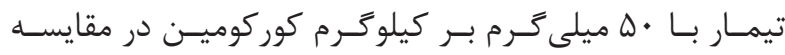

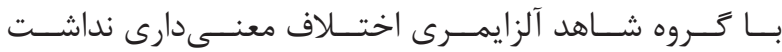

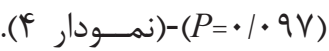

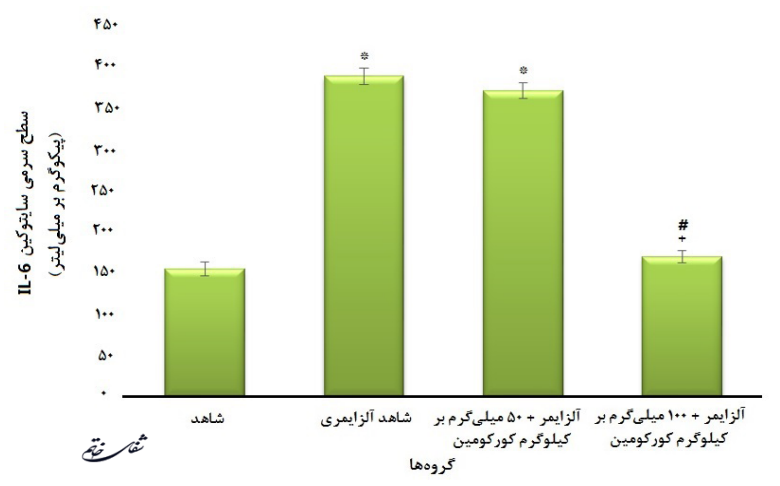

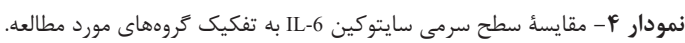

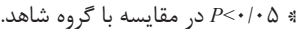

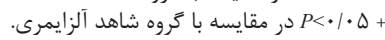

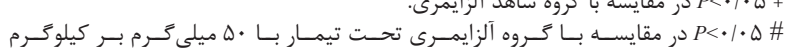
كوركوميـن.

بحث و نتيجه تيرى

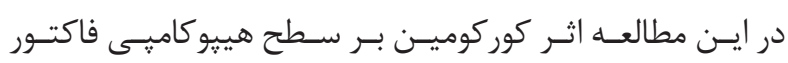

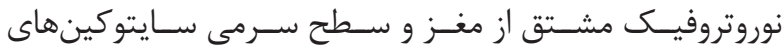

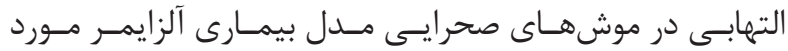

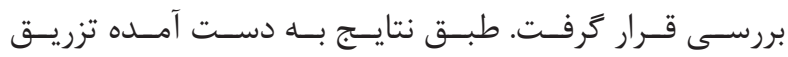

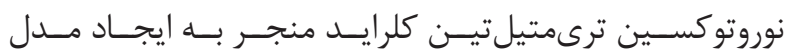

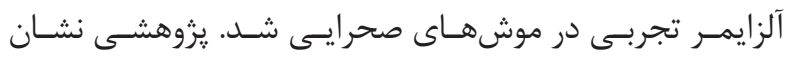

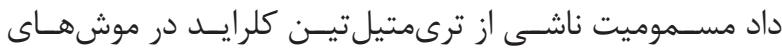

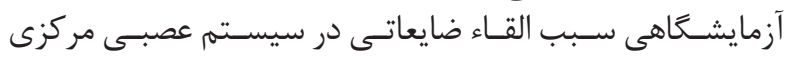

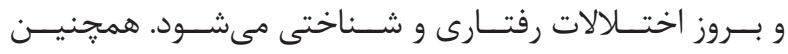

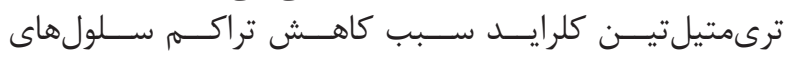

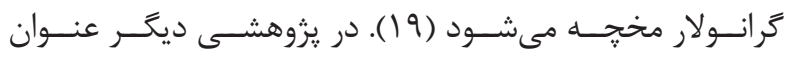

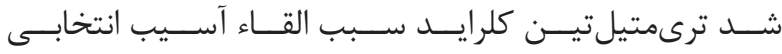

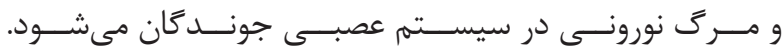

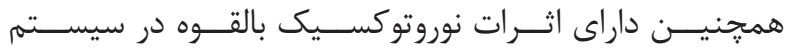

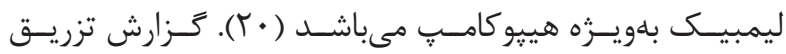

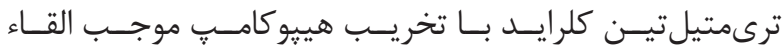

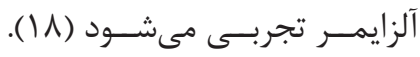

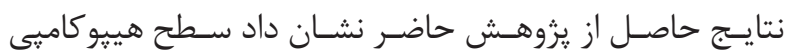

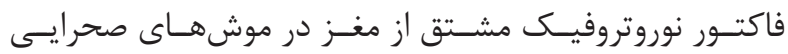

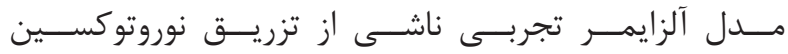

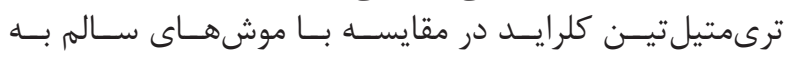

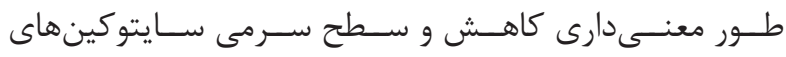




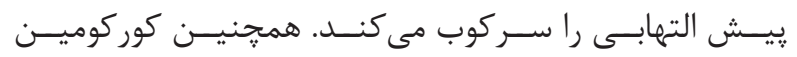

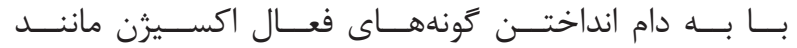

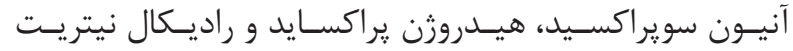

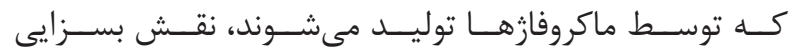

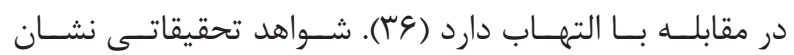

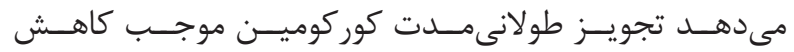

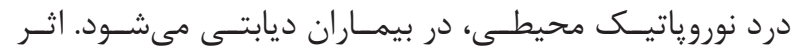

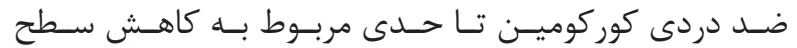

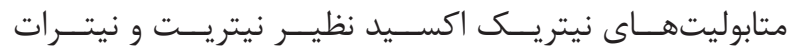

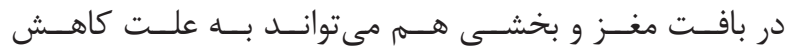

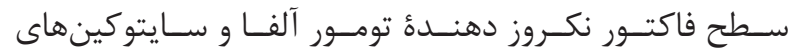

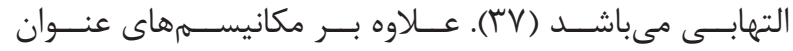

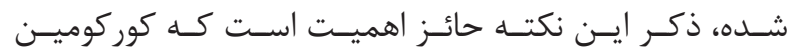

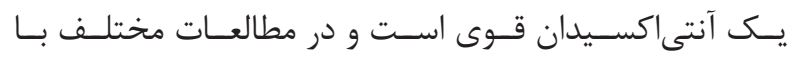

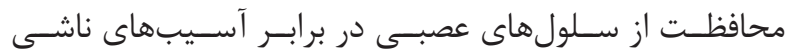

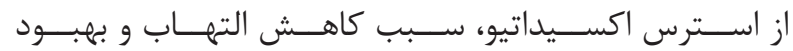

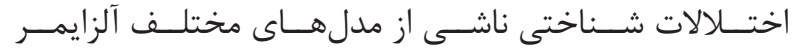

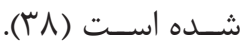

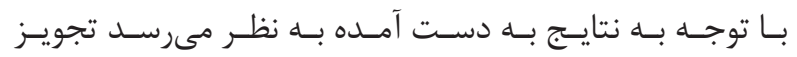

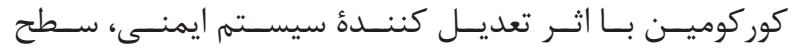

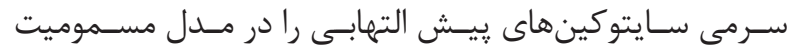

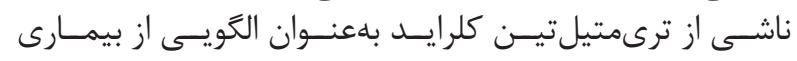

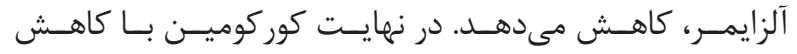

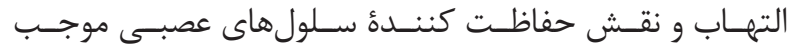

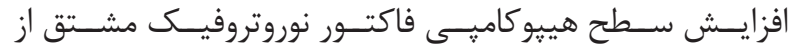

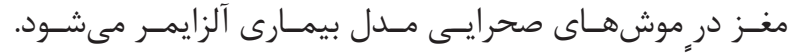

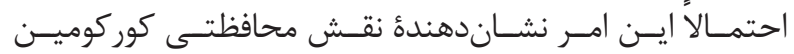

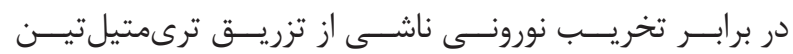

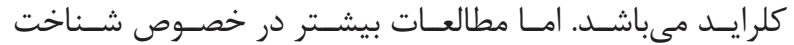

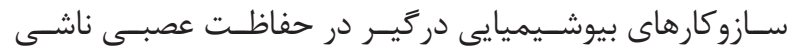

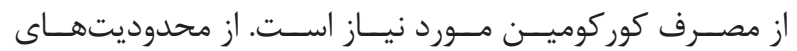

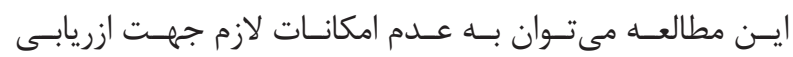

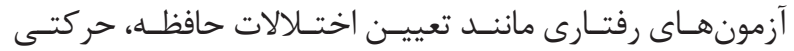

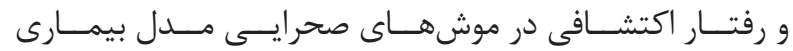

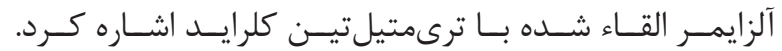

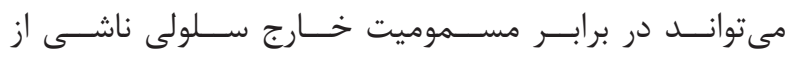

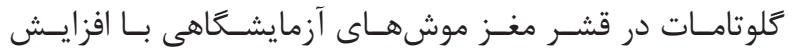

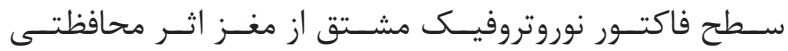

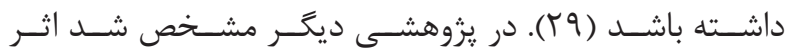

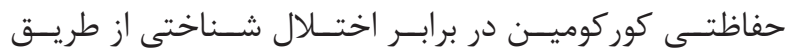

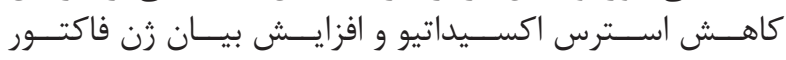

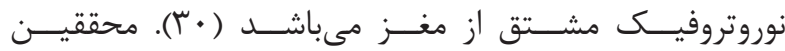

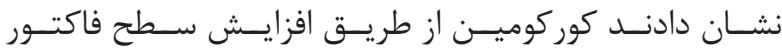

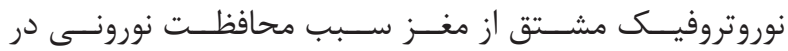

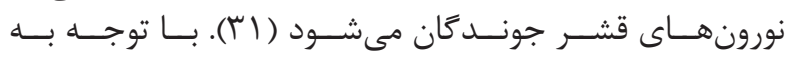

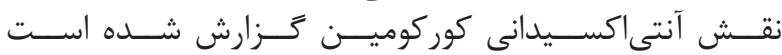

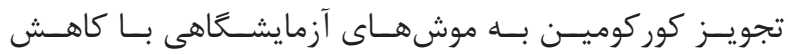

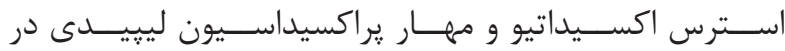

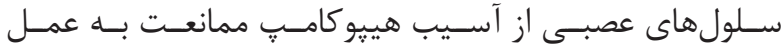

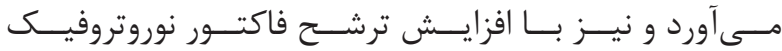

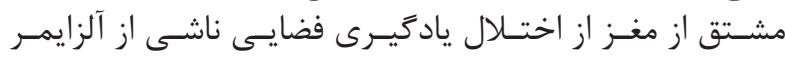

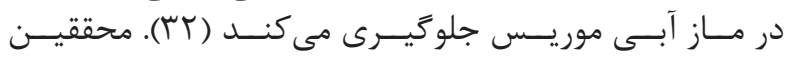

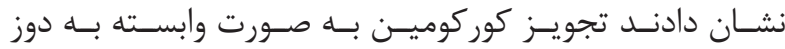

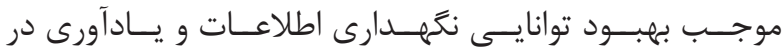

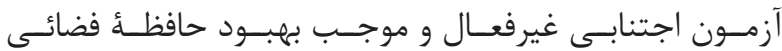

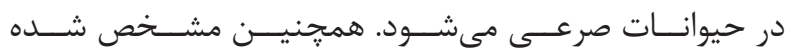

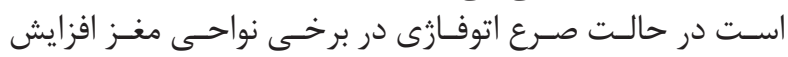

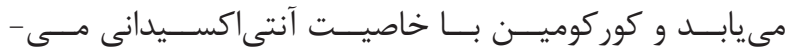

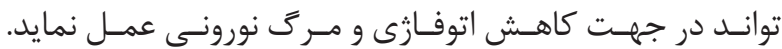

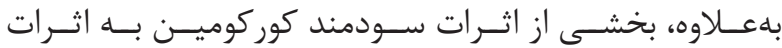

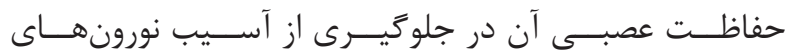

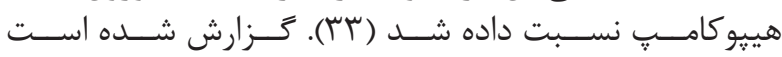

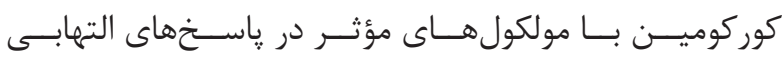

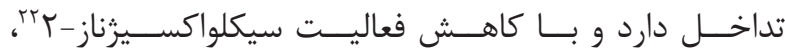

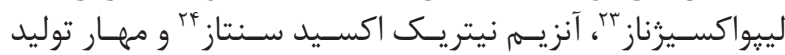

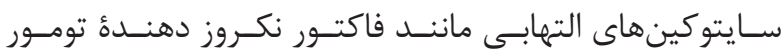

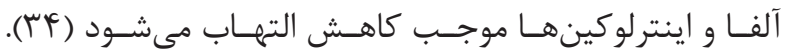

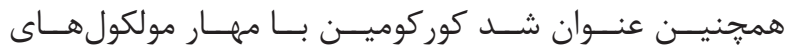

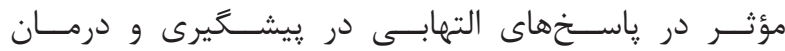

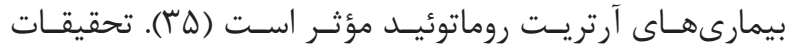

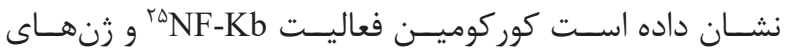

\footnotetext{
${ }^{22}$ Cyclooxygenase- 2

${ }^{23}$ Lipoxygenase

${ }^{24}$ Nitric oxide synthase

${ }^{25}$ Nuclear factor kappa-light-chain-enhancer of activated B cells
} 
1. Cacace R, Sleegers $\mathrm{K}$, Van Broeckhoven $\mathrm{CH}$. Molecular genetics of early-onset Alzheimer's disease revisited. Alzheimers Dement. 2016; 12(6): 733-48.

2. Dicou E. Neurotrophins and neuronal migration in the developing rodent brain. Brain Res Rev. 2009; 60(2): 408-17.

3. Allen SHJ, Watson JJ, Shoemark DK, Barua NU, Patel NK. GDNF, NGF and BDNF as therapeutic options for neurodegeneration. Pharmacol Ther. 2013; 138(2): 155-75.

4. Zhang F, Kang ZH, Li W, Xiao ZH, Zhou X. Roles of brain-derived neurotrophic factor/tropomyosin-related kinase B (BDNF/TrkB) signalling in Alzheimer's disease. J Clin Neurosci. 2012; 19(7): 946-9.

5. Stenken JA, Poschenrieder AJ. Bioanalytical chemistry of cytokines - a review. Analytica Chimica Acta. 2015; 853: 95-115.

6. Cheng X, Shen Y, Li R. Targeting TNF: a therapeutic strategy for Alzheimer's disease. Drug Discov Today. 2014; 19(11): 1822-7.

7. Fedotova J, Soultanov V, Nikitina T, Roschin V, Ordyan N, Hritcu L. Cognitive-enhancing activities of the polyprenol preparation Ropren ${ }^{\circledR}$ in gonadectomized $\beta$-amyloid (25-35) rat model of Alzheimer's disease. Physiol Behav. 2016; 157: 55-62.

8. Kosaraju J, Madhunapantula SV, Chinni S, Khatwal RB, Dubala A, Muthureddy Nataraj SK, et al. Dipeptidyl peptidase-4 inhibition by Pterocarpus marsupium and Eugenia jambolana ameliorates streptozotocin induced Alzheimer's disease. Behav Brain Res. 2014; 267: 55-65.

9. Corvino V, Marchese E, Giannetti S, Lattanzi W, Bonvissuto D, Biamonte F, et al. The neuroprotective and neurogenic effects of neuropeptide $\mathrm{Y}$ administration in an animal model of hippocampal neurodegeneration and temporal lobe epilepsy induced by trimethyltin. J Neurochem. 2012; 122(2): 415-26.

10. Bhat AH, Dar KB, Anees S, Zargar MA, Masood A, Sofi MA, et al. Oxidative stress, mitochondrial dysfunction and neurodegenerative diseases; a mechanistic insight. Biomed Pharmacother. 2015; 74: 101-10.

11. Jiang T, Sun Q, Chen SH. Oxidative stress: a major pathogenesis and potential therapeutic target of antioxidative agents in Parkinson's disease and Alzheimer's. Prog Neurobiol. 2016; 147: 1-19.

12. Maheshwari RK, Singh AK, Gaddipati J, Srimal
RC. Multiple biological activities of curcumin. Life Sci. 2006; 78(18): 2081-7.

13. Kant V, Gopal A, Pathak NN, Kumar P, Tandan SK, Kumar D. Antioxidant and anti-inflammatory potential of curcumin accelerated the cutaneous wound healing in streptozotocin-induced diabetic rats. Int Immunopharmacol. 2014; 20(2): 322-30.

14. Yang F, Lim GP, Begum AN, Ubeda OJ, Simmons MR, Ambegaokar SS. et al. Curcumin inhibits formation of amyloid beta oligomers and fibrils, binds plaques, and reduces amyloid in vivo. J Biol Chem. 2005; 280(7): 5892-901.

15. Pan R, Qiu S, Lu DX, Dong J. Curcumin improves learning and memory ability and its neuroprotective mechanism in mice. Chin Med J (Engl). 2008; 121(9): 832-9.

16. Tehranipour M, Khayyatzade J, Javaheri fard R. The protective effects of curcuma longa total extract on spinal cord neuroglia cell degeneration after the sciatic nerve compression in rats. AMUJ. 2010; 13(1): 83-9.

17. Kowluru RA, Kanwar M. Effects of curcumin on retinal oxidative stress and inflammation in diabetes. Nutr Metab (Lond). 2007; 4: 8. Doi: 10.1186/1743-7075-4-8.

18. Bazyar Y, Rafiei S, Hosseini A, Edalatmanesh M A. Effect of endurance exercise training and gallic acid on tumor necrosis factor- $\alpha$ in an animal model of Alzheimer's disease. Shefaye Khatam. 2015; 3(3): 21-6.

19. Lee S, Yang M, Kim J, Son Y, Kim J, Kang S. et al. Involvement of BDNF/ERK signaling in spontaneous recovery from trimethyltin-induced hippocampal neurotoxicity in mice. Brain Res Bull. 2016; 121: 48-58.

20. Earley B, Burke M, Leonard BE. Behavioural, biochemical and histological effects of trimethyltin (TMT) induced brain damage in the rat. Neurochem Int. 1992; 21(3): 351-66.

21. Corvino V, Marchese E, Michetti F, Geloso MC. Neuroprotective strantegics in hippocampal neuro degeneration induced by the neurotoxicant trimethylyin. Neurochem Res. 2013; 38(2): 240-53.

22. O'Connell A, Earley B, Leonard BE. The neuroprotective effect of tacrine on trimethyltin induced memory and muscarinic receptor dysfunction in the rat. Neurochem Int. 1994; 25(6): 555-66.

23. Lee S, Yang M, Kim J, Kang S, Kim J, Kim JC, et al. Trimethyltin-induced hippocampal neurodegeneration: a mechanism-based review. Brain Res Bull. 2016; 125: 
187-99.

24. Maier WE, Brown HW, Tilson HA, Luster MI, Harry GJ. Trimethyltin increases interleukin (IL)-l $\alpha$, IL-6 and tumor necrosis factor $\alpha$ mRNA levels in rat hippocampus. J Neuroimmunol. 1995; 59(1-2): 65-75.

25. Licastroa F, Pedrinia S, Caputob L, Annonib G, Davisa LJ, Ferric C, et al. Increased plasma levels of interleukin-1, interleukin-6 and $\alpha$-1-antichymotrypsin in patients with Alzheimer's disease: peripheral inflammation or signals from the brain? J Neuroimmunol. 2000; 103(1): 97-102.

26. Han S, Park J, Mook-Jung I. Amyloid $\beta$-interacting partners in Alzheimer's disease: from accomplices to possible therapeutic targets. Prog Neurobiol. 2016; 137 : 17-38.

27. Stewart CR, Stuart LM, Wilkinson K, Van Gils JM, Deng J, Halle A, et al. CD36 ligands promote sterile inflammation through assembly of a Toll-like receptor 4 and 6 heterodimer. Nat Immunol. 2010; 11(2): 155-61.

28. Hosseinzadeh S, Dabidi Roshan V. Effects of Curcumin supplementation on BDNF and oxidative/ antioxidative process in rat's hippocampus which exposed to lead. J Gorgan Uni Med Sci. 2011; 13(2): $1-8$.

29. Wu A, Ying Z, Gomez-Pinilla F. Dietary curcumin counteracts the outcome of traumatic brain injury on oxidative stress, synaptic plasticity, and cognition. Exp Neurol. 2006; 197(2): 309-17.

30. Wang R, Li YB, Li YH, Xu Y, Wu HL, Li XJ. Curcumin protects against glutamate excitotoxicity in rat cerebral cortical neurons by increasing brain-derived neurotrophic factor level and activating TrkB. Brain Res. 2008; 1210: 84-91.

31. Wang R, Li YH, Xu Y, Li YB, Wu HL, Guo H, et al.
Curcumin produces neuroprotective effects via activating brain-derived neurotrophic factor/TrkB-dependent MAPK and PI-3K cascades in rodent cortical neurons. Prog Neuropsychopharmacol Biol Psychiatry. 2010; 34(1):147-53.

32. Liu D, Wang Z, Gao Z, Xie K, Zhang Q, Jiang H, et al. Effects of curcumin on learning and memory deficits, BDNF, and ERK protein expression in rats exposed to chronic unpredictable stress. Behav Brain Res. 2014; 271: 116-21.

33. Anovadiya1 AP, Sanmukhani JJ, Vadgama VK, Tripathi CB. Evaluation of antiepileptic and memory retention activity of curcumin per se and in combination with antiepileptic drugs. Asian J Pharm Clin Res. 2013; 6(2): $145-8$.

34. Jurenka JS. Anti-inflammatory properties of curcumin, a major constituent of Curcuma longa: a review of preclinical and clinical research. Altern Med Rev. 2009; 14(2): 141-53.

35. Mateen S, Moin S, Zafar A, Khan AQ. Redox signaling in rheumatoid arthritis and the preventive role of polyphenols. Clin Chim Acta. 2016; 463: 4-10.

36. Li W, Suwanwela NC, Patumraj S. Curcumin by down-regulating NF-kB and elevating Nrf2, reduces brain edema and neurological dysfunction after cerebral I/R. Microvasc Res. 2016; 106: 117-27.

37. Banafshe HR, Hamidi GA, Noureddini M, Mirhashemi SM, Mokhtari R, Shoferpour M. Effect of curcumin on diabetic peripheral neuropathic pain: possible involvement of opioid system. Eur J Pharmacol. 2014; 723: 202-6.

38. Ghosh S, Banerjee S, Sil PC. The beneficial role of curcumin on inflammation, diabetes and neurodegenerative disease: a recent update. Food Chem Toxicol. 2015; 83: 111-24. 